\title{
Micromanipulation intracellulaire par pinces optiques
}

A. Ott

Laboratoire de Physico Chimie Curie (PCC), UMR 168 du CNRS, Institut Curie, Recherche, 11 rue Pierre et Marie Curie, 75231 Paris cedex 05, France

\begin{abstract}
Résumé : Les pinces optiques consistent en un faisceau laser focalisé par un objectif adapté. Ils permettent ainsi de piéger et de manipuler certains objets sous un microscope. Ils constituent la seule technique de manipulation intracellulaire non-invasive. Nous donnons quelques exenples de leur application dans le domaine.
\end{abstract}

Les pinces optiques ['] consistent en un faisceau lumineux focalisé par l'objectif d'un microscope ayant une ouverture numérique très grande. On crée ainsi une force sur des particules ayant un indice de réfraction plus élevé que le milieu environnant. Elle est due au gradient de champ et au transfert de moment de la lumière réfractée. Le bilan des forces en présence assure un piégeage dans le plan focal de particules de tailles comparables à la longueur d'onde (en pratique de 0,2 à quelque dizaines de microns). Il est alors possible de déplacer les objets ainsi piégés. La force de piégeage appliquée par la pince optique peut être calibrée et utilisée pour mesurer les forces mises en jeu dans différents processus biologiques, dans une échelle de 0.1 à environ une centaine de picoNewtons. Une approximation heuristique de cette force est donnée par

$$
\mathrm{F}=0.03(\mathrm{nP} / \mathrm{c})(1)
$$

ou $n$ est la différence d'indice, $c$ la vitesse de la lumière et $P$ est la pression de radiation de la lumière. $\mathrm{P}=\mathrm{y} \mathrm{I} / \mathrm{c}$ ou I est la densité de puissance de la lumière, $\mathrm{y}=1$ pour une absorption parfaite et $y=2$ pour une réflexion idéale. Des équations plus précises se trouvent dans la référence $\left[{ }^{t}\right]$. En ajoutant un détecteur de position la pince optique peut être utilisée dans un mode asservi et des mesures de force isométriques peuvent être effectuées.

Les pinces optiques ont non seulement permis de mesurer, à l'échelle du nm et du $\mathrm{pN}$, le pas élémentaire du moteur moléculaire actine-myosine ainsi que la force générée par un seul moteur $\left.{ }^{3}\right]$, mais aussi elles ont été utilisées pour la manipulation à l'échelle cellulaire $\left.{ }^{\ddagger}\right]$. Parmi ces dernières on peut citer le déplacement de bactéries conduisant à la mesure de la force générée par leur moteur flagellaire $\left[{ }^{5}\right]$. Ashkin et Dziedzic $\left[{ }^{6}\right]$ ont mesuré des propriétés viscoélastiques à l'intérieur de cellules végétales vivantes avec une pince optique. Ils ont également réussi à déterminer la force générée par le transport d'organelles le long de microtubules in vivo $\left[{ }^{7}\right]$. Une perspective nouvelle est apparue avec l'expérience de Berns $\left[{ }^{8}\right]$ qui a réussi à transporter des chromosomes isolés de part et d'autre du faisceau mitotique. Ceci a permis de mettre en évidence une régulation mécanique du transport de chromosomes lors de la mitose.

Notons que les pinces optiques constituent la seule technique de manipulation intracellulaire non invasive.

\section{Références}

'Ashkin R. L. and Storb, R. Science 210 (1980) 1081-10888;

Ashkin A., Dziedzic, J. M. Science 235 (1987) 1517-1520.

${ }^{2}$ Block S. M., Goldstein L. S. B., et Schnapp B. J. Nature 348 (1990) 348-352; 
Block S. M., Blair D. F. et Berg H. C. Nature 338 (1990) 514-518.

? Finer J.T., Simmons R. M., Spudich J. A. Nature 368 (1994) 113-119.

${ }^{4}$ pour une revue voir Weber G., Greulich K. O. Int. Rev. Cytol. 133 (1992) 1-41.

${ }^{5}$ Block S. M., Blair D. F., Berg H. C. Nature 338 (1989) 514-518.

${ }^{6}$ Ashkin A., Dziedzic, J. M.Proc. Natl. Acad. Sci. 86 (1989) 7914-7918.

7 Ashkin A. Schutze K. Dziedzic J. M. Euteneuer U. Schliwa M. Nature 348 (1990) 346-348.

8 Berns M. W. Wright W. H., Tromberg B. J., Profeta G. A., Andrews J. J., Walter R. J. Proc. Natl. Acad. Sci. 86 (1989) 4539-4543;

Liang H. Wright W. H. , He W., Berns M. W. Exp. Cell Res 197 (1991) 21-35. 